\title{
ARTICLES
}

\section{Skin Conductance Changes During the First Year of Life in Full-Term Infants}

\author{
KNUT G. HERNES, LARS MØRKRID, ASBJØRN FREMMING, STEIN ØDEGÅRDEN, \\ ØRJAN G. MARTINSEN, HANNE STORM \\ Department of Paediatric Research, National Hospital, Oslo, Norway [K.G.H., H.S.], Department of \\ Clinical Chemistry and Department of Obstetrics and Gynaecology, National Hospital, Oslo, Norway \\ [L.M.], Department of Physics, University of Oslo, Norway [A.F., S.Ø., Ø.G.M.]
}

\begin{abstract}
ABSTR
Skin conductance changes (SCC) reflect the activity in the
sympathetic postganglionic cholinergic fibers, which innervate
the sweat glands located in the palm of the hand and the sole of
the foot. The purpose of this study was to measure the changes
in the number of waves per second, the wave amplitude, and the
mean skin conductance level during the 1 st year of life. During
SCC elicited by an auditory stimulus we measured the percent-
age of infants that responded, the amplitude, latency and recov-
ery times, and any habituation pattern. Thirty-nine full-born,
healthy infants were investigated during their 1 st and 3rd days of
life, at 3 and 10 wk of life, and at 6 and 12 mo of life. The mean
skin conductance level ( $p<0.001)$, the number of waves with an
amplitude threshold higher than 0.5 and $1.0 \mu$ siemens $(p<$
0.001), and the amplitude of the waves $(p<0.001)$ all increased
\end{abstract}
The sympathetic nervous system innervates a large number of end organs in the human body, such as the emotional sweat glands in the palm of the hand and sole of the foot, the blood vessels in the skin and muscles, the adrenal glands, and the heart. Wallin et al. have shown that although the sympathetic nervous system normally acts as a single system, parts of it can also be separately activated (1). A direct relation has been shown in humans between CNS norepinephrine release, sampled in internal jugular venous plasma, and the peripheral sympathetic nerve-firing rate (2). The activity in the sympathetic fibers of the nerves supplying the different organs has been investigated with the microelectrode recording technique (3). A direct correlation was found between the skin sympathetic firing rate, and also the firing amplitude in the innervating nerves, on the one hand and the increased sweat response

Received July 26, 2000; Accepted February 22, 2002.

Correspondence: Knut G Hernes, M.D., Department of Paediatric Research, National Hospital, Oslo, Norway; e-mail: kshernes@hotmail.com

Supported by The SIDS Society, Oslo, Norway.

DOI: 10.1203/01.PDR.0000036879.07350.12 during the first $10 \mathrm{wk}$ of life. The percentage that responded to stimuli increased from $8 \%$ to $50 \%$, and the amplitude of the response increased during the first $10 \mathrm{wk}$ of life $(p<0.001)$. The level of arousal influenced the mean skin conductance level, the number of waves per second, and the amplitude of the waves during the 1st year of life. In conclusion, these results indicate that the part of the sympathetic nervous system associated with arousal develops during the first 10 wk of life. (Pediatr Res 52: 837-843, 2002)

Abbreviations
SCC, skin conductance changes
HRV, heart rate variability
$\boldsymbol{\mu S}$, microsiemens

located in the sweat glands in the palms of the hands and soles of the feet, measured in terms of the number and amplitude of spontaneous skin conductance waves, on the other $(3,4)$.

The sweat gland response itself can be measured both in terms of skin evaporation and in terms of skin electrical admittance, as well as by visual inspection using a microscope. The real part of skin admittance, the skin conductance changes (SCC), can be measured during spontaneous activity and during responses elicited by external stimulation. Skin conductance reflects the level of readiness of the nervous system, or cortical vigilance, and may be altered by the infant's state of arousal. SCC may be due to activity in areas of the CNS such as the brainstem, reticular substance, the hypothalamus, the premotoric cortex, the amygdala, the hippocampus and sympathetic cervical ganglions (5).

The purpose of this study was to investigate the development of that part of the sympathetic nervous system associated with arousal during the 1 st year of life by measuring the emotional sweat gland response in terms of spontaneous and stimulated SCC. The mean skin conductance level, the number of waves 
per second, and the amplitude of the waves were measured during spontaneous SCC. The percentage of infants that responded, the amplitude of the response, the latency and recovery times, and any signs of habituation were also measured during SCC elicited by auditory stimulation. Our method uses an alternating current for the detection of the SCC.

\section{METHOD}

Subjects. The infants were all recruited from The Department of Obstetrics and Gynaecological at the National Hospital, Oslo, Norway. A total of 56 newborn infants were enrolled in the study, 29 females and 27 males. Eight of these (5 male, 3 female) dropped out of the study, and were subsequently excluded. Four infants (all male) moved to different parts of the country and were also excluded together with 5 infants (4 male, 1 female) that missed 3 or more recordings. The rest, a total of 14 males and 25 females, completed the study. All infants were born at $>37$ wk of gestation, based on the delivery date determined by ultrasound. The infants were all healthy and had an Apgar score equal to or better than 9 at $5 \mathrm{~min}$. All the parents were of Scandinavian origin. Ten adults, aged 21 to $56 \mathrm{y}, 5$ male, 5 female, were also recruited to be able to compare the level of SCC in adults with that in the infants. Information about the delivery was recorded, including the method of delivery (vaginal, caesarean or breech delivery) and any delivery aid (suction, forceps) or anesthetics used. The SCC was measured between 10.00 and 17.00. Informed consent was obtained, and the regional Ethics Committee for Human Studies approved the protocol.

Apparatus. Conductance was measured essentially with the method developed by Qiao et al. (6), but with the use of only one excitatory frequency, $88 \mathrm{~Hz}$. Conductance was chosen in preference to resistance because of the parallel nature of the electrical polarization and conduction in the skin (7). Thus low frequency electrical conductance reflects ionic conduction in the stratum corneum, which is largely determined by sweat duct filling $(8,9)$. An important point that is often confused in the literature is that electrical resistance is the inverse of conductance only in the case of direct current. Alternating current resistance is a function of both conductance and susceptance. This means that the choice of conductance rather than resistance is crucial. A frequency of $88 \mathrm{~Hz}$ is high enough to significantly reduce the requirements for low electrode polarizability, but low enough to ensure minimal influence from viable skin (9).

An applied voltage of $50 \mathrm{mV}$ and a three-electrode system were used to allow unipolar measurement (10). The threeelectrode system consists of a measuring electrode (M), a counter current electrode (C) and a reference voltage electrode (R), which ensure a constant applied voltage across the stratum corneum beneath the $\mathrm{M}$ electrode. The resulting current through the $\mathrm{M}$ electrode was then converted to a voltage in a transresistance amplifier, and a synchronous rectifier was used to decompose the signal into conductance and susceptance.

In this study we only investigated the conductance. An optical coupler was developed in conformity with the safety regulations in IEC-60-601. A loudspeaker producing broad- spectrum white sound of $2 \mathrm{~s}$ duration, with an effect of approximately $75 \mathrm{~dB}, 30 \mathrm{~cm}$ away from the subject's ear, was used as an external stimulant. Beckman electrodes were used for the recording (Sensorimedics no. 650418). The electrodes were fixed to the skin by disks of double-sided adhesive tape produced by $3 \mathrm{M}$, MN, U.S.A. Conductive paste from the National Hospital Pharmacy, containing 6 g hydroxyethylcellulose $700,0.58 \mathrm{~g} \mathrm{NaCl}, 0.1 \mathrm{~g}$ methylparahydroxybenzene, $0.1 \mathrm{~g}$ propylparahydroxybenzene, $2 \mathrm{~g}$ alcohol $96 \%$, and purified water up to $100 \mathrm{~g}$ was used to improve electrode conductance.

Software program. Skin conductance was sampled with a frequency of $50 \mathrm{~Hz}$, with 12-bit resolution. The data were stored online using a portable IBM-compatible computer and were analyzed off line by means of a software package. The skin conductance recording and analysis software program were custom made using the LABVIEW program, National Instruments, U.S.A. (11). The amplitude threshold, slope threshold of the waves, latency time window, and recovery time window were all adjustable. The $0.02 \mu$ siemens amplitude threshold was chosen after analyzing the magnitude of the electrical noise. Higher thresholds were set at 0.5 and 1.0 $\mu$ siemens to identify the number of waves with higher amplitudes. The threshold for the slope, defined as the mean distance from the valley to the peak/time to reach peak, was set at 2 $\mu$ siemens/s, and excluded the artifacts with a steeper slope. The method produced artifacts when an electrode was disconnected from the skin but was not sensitive to movement or changes in the normal temperature range. During the auditory measurements the latency time window was set at 0.5 to $5 \mathrm{~s}$. The software program was designed to detect the recovery time after a response, defined as $50 \%$ recovery of the response amplitude. All responses were analyzed $5 \mathrm{~s}$ before, and $15 \mathrm{~s}$ after the auditory stimuli.

During spontaneous SCC, the software program measured and analyzed the mean spontaneous skin conductance level, the number of waves per second, and the amplitude of the waves (Fig. 1). During SCC elicited by auditory stimuli the software program used coherent averaging to identify whether or not the infant responded to the sound given. This method superimposes all the responses and eliminates the spontaneous waves. This allows it to calculate the mean reaction to the stimuli (Fig. 2). A positive response was defined as a wave with an amplitude threshold higher than $0.02 \mu$ siemens. The software program analyzed the amplitude of the response, the percentage of infants that responded and the latency and recovery times. It also calculated the latency time, amplitude and recovery time for each individual response to detect any pattern of habituation. Both hardware and software are developed by Med-Storm Innovation, product number 060895, Oslo, Norway.

Procedure. Measurements were made at the following time points: during the 1 st and 3rd day of life, the 3rd and 10th weeks of life, and at 6 and 12 mo of age - time points $1,2,3,4,5$ and 6 respectively. The $\mathrm{C}$ electrode was placed on the medial side of the foot over the abductor hallucis muscle on the plantar surface. The $\mathrm{M}$ electrode was placed directly beneath the ankle, and the $\mathrm{R}$ electrode was placed on the dorsal side of the foot. These areas were chosen in 


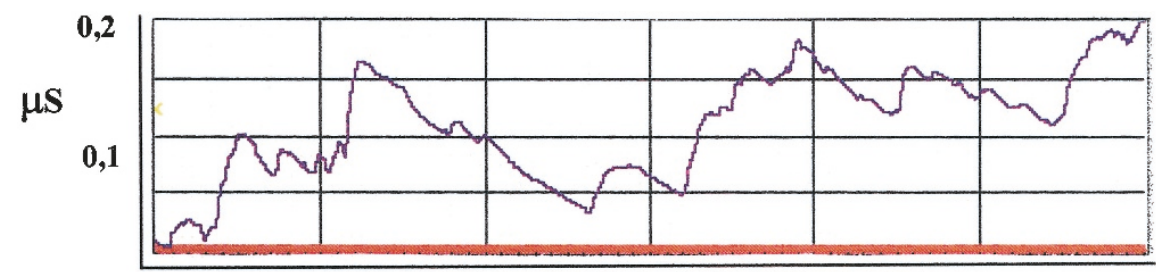

seconds

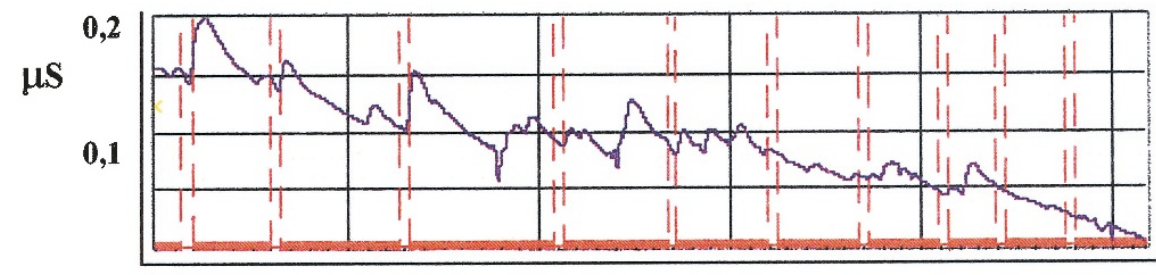

seconds

Figure 1. Spontaneous skin conductance changes (top). The mean skin conductance level, the number of waves per second and the amplitude of the waves were measured with the infants in a prone and a supine position. Skin conductance changes during auditory stimulation (bottom). A total of 10 auditory stimuli were administered over a period of $4 \mathrm{~min}$. The vertical double line represents the stimuli.

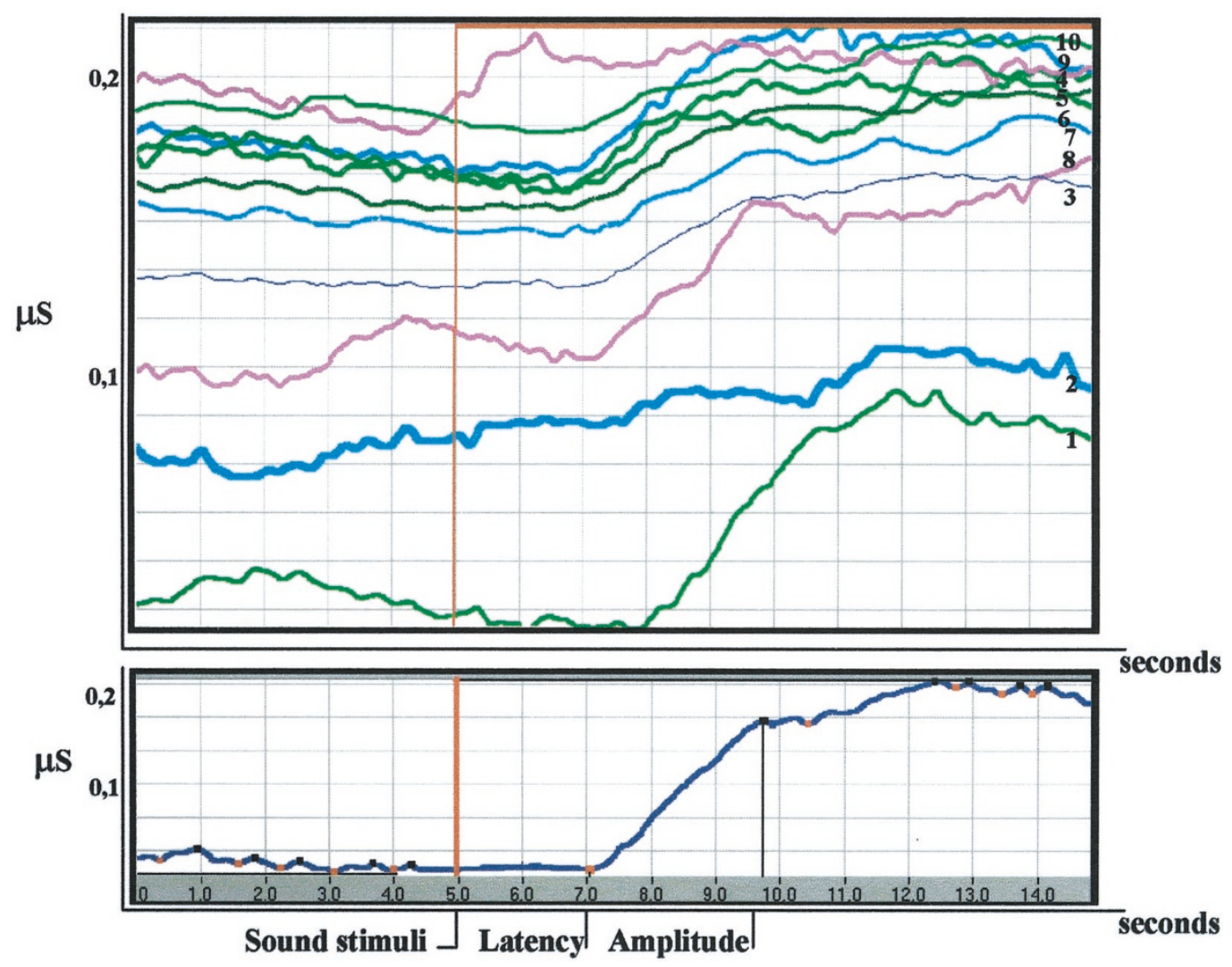

Figure 2. Coherent averaging was used to analyze the response during the skin conductance changes to auditory stimuli. In the top diagram, all 10 responses to auditory stimuli administered to a particular infant are recorded (numbers 1-10). In the lower diagram the smoothed average of all 10 measurements is presented. All the peaks and valleys were identified. A sound stimulus initiated a response with a maximum amplitude after a latency time, but no recovery was seen within $15 \mathrm{~s}$

accordance with the Edelberg guidelines for placement of electrodes to obtain the most sensitive measurement (12). Each subject was placed on an examination table, and spontaneous SCC was recorded for $3 \mathrm{~min}$ in a prone and 3 min in a supine position, with a random starting position. After the spontaneous SCC measurement, SCC during auditory stimulation was recorded. During this period the subjects were exposed to ten auditory stimuli, after 0,25 ,
$60,100,130,155,180,200,215$, and 235 s. A habituation pattern was defined as three nonresponses after a response pattern. During each measurement the infant's temperature, clinical arousal, and time since the last feed were all recorded. The level of arousal was divided into 4 different categories: 1 = asleep, 2 = awake, eyes open, 3 = awake, active body movements, $4=$ screaming intensely with vigorous body movements (12). 
Statistics. The statgraphics statistical package was used. Parametric tests were used for variables that were normally distributed, if necessary after transformation. The mean skin conductance level had to be log-transformed to fulfill the criteria. An ANOVA was used to test for group differences, and a post hoc test was subsequently applied to identify where the significant differences were located. The degree of habituation was measured by calculating the nonparametric Spearman correlation coefficient between the number of stimuli and wave amplitude.

Multivariable analysis was used to identify any effects of the infant's position, arousal, temperature, sex, and the time elapsed since the last feed (defined as less or more than $60 \mathrm{~min}$ ) during the spontaneous skin conductance changes. The model was also corrected for the effect of time, by introducing time as an independent factor. The effects of delivery aids and method of delivery were identified by multivariable analysis. No statistical comparisons were made between the infant and the adult populations. The significance level was 0.05 .

\section{RESULTS}

There were no statistical differences between the results from the measurements in a prone and a supine position, so only the results from the supine position are presented.

Spontaneous SCC. The mean $( \pm \mathrm{SD})$ level of arousal during the spontaneous measurements was $2.12( \pm 0.83)$ during the first measurement, $2.5( \pm 1.03)$ during the second, 2.71 $( \pm 0.77)$ during the third, $2.76( \pm 0.59)$ during the fourth, 2.54 $( \pm 0.49)$ during the fifth and $2.30( \pm 0.45)$ during the last measurement.

The mean skin conductance level. The mean skin conductance level increased significantly during the first $10 \mathrm{wk}$ of life, from $2.63 \mu$ siemens on day 1 to $3.67 \mu$ siemens at $10 \mathrm{wk}$ of life $(p<0.001)$. (The adult value was $1.03 \mu$ siemens (Fig. 3).) The results from measurements 1,2 , and 3 were significantly lower than the results from measurements 4, 5, and 6. The ANOVA model for the mean skin conductance level was positively

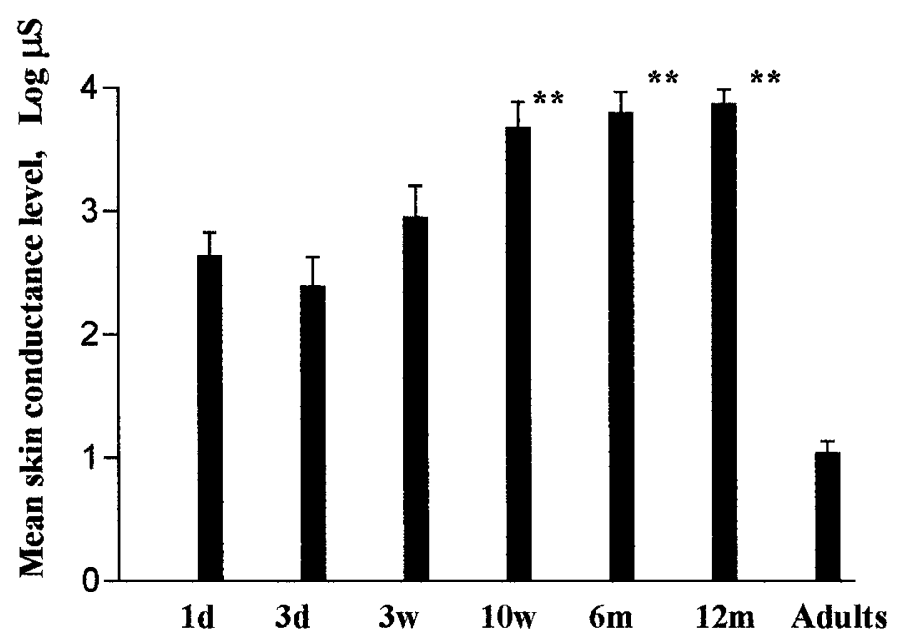

Figure 3. The mean skin conductance level, measured in the logarithm of $\mu$ siemens. The measurements were made during the 1 st day of life $(1 \mathrm{~d})$, the 3rd day of life $(3 \mathrm{~d})$, and at three weeks $(3 \mathrm{w}), 10 \mathrm{wk}(10 \mathrm{w})$, six months $(6 \mathrm{~m})$, and $12 \mathrm{mo}(12 \mathrm{~m}) .(* * p<0.01)$ correlated to the arousal $(p<0.001)$, but when correcting for the effect of time points, arousal did not show a significant additional effect. The time since the last feed had no effect on the mean skin conductance level.

The number of waves. A significant decrease in the mean number of smaller waves, defined by an amplitude threshold of $0.02 \mu$ siemens per second (Fig. 4), was found between the 3rd day of life ( 0.42 waves per second) and the end of the 1st year ( 0.19 waves per second) $(p<0.001)$. (The adult value was 0.25 waves per second.) Result from measurement 1 was significantly different from the results from measurements 2 and 3. Results from measurements 2 and 3 were different from the results from measurements 5 and 6 , and the results from measurement 4 was different from the result from measurement $6(p<0.001)$.

At amplitude thresholds of 0.5 and $1.0 \mu$ siemens, a significant increase in the mean number of waves was seen during the first 10 wk of life $(p<0.001)$ (Fig. 4). Results from measurements 1 and 2 were different from the results from measurements 4, 5 and 6 , and the result from measurement 3 was different from the results from measurements 4 and 5 . These results indicate that the low-amplitude waves decreased as the high-amplitude waves increased during the 1st year of life. At amplitude thresholds 0.02, 0.5 and $1.0 \mu$ siemens, the number of waves per second during the 1 st year of life was positively correlated with the infants' level of arousal $(p<$ 0.01 ). Correcting for the effect of time, the number of waves per second still proved to be positively correlated with the infant's arousal, using the threshold of $0.5 \mu$ siemens as well as $1.0 \mu$ siemens $(p<0.05)$. The feeding time had no effect on the number of waves per second.

The amplitude of the waves. The mean amplitude, using an amplitude threshold of $0.02 \mu$ siemens $(p<0.001)$ (Fig. 5), increased significantly during the first 6 mo of life, from 0.23 $\mu$ siemens during the 1st day of life up to $1.26 \mu$ siemens at 6 mo of life, and especially during the first $10 \mathrm{wk}$ of life. The results from measurements 1,2 , and 3 were different from the results from measurements 4,5 , and 6 . (The adult value was $0.25 \mu$ siemens.) At amplitude thresholds of 0.5 and $1.0 \mu$ siemens a significant increase during the first $10 \mathrm{wk}$ of life was

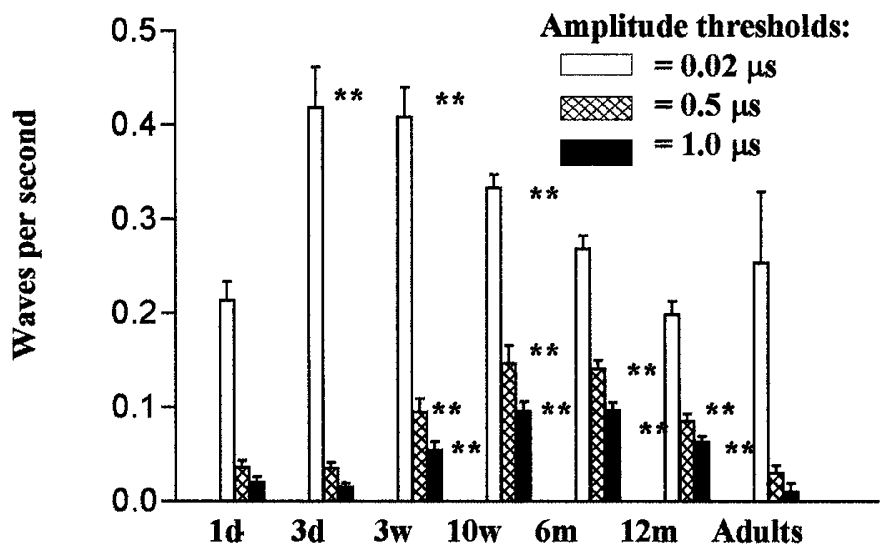

Figure 4. The number of waves per second during spontaneous skin conductance changes, using amplitude thresholds of $0.02,0.5$ and $1.0 \mu$ siemens $(* * p$ $<0.01)$. 


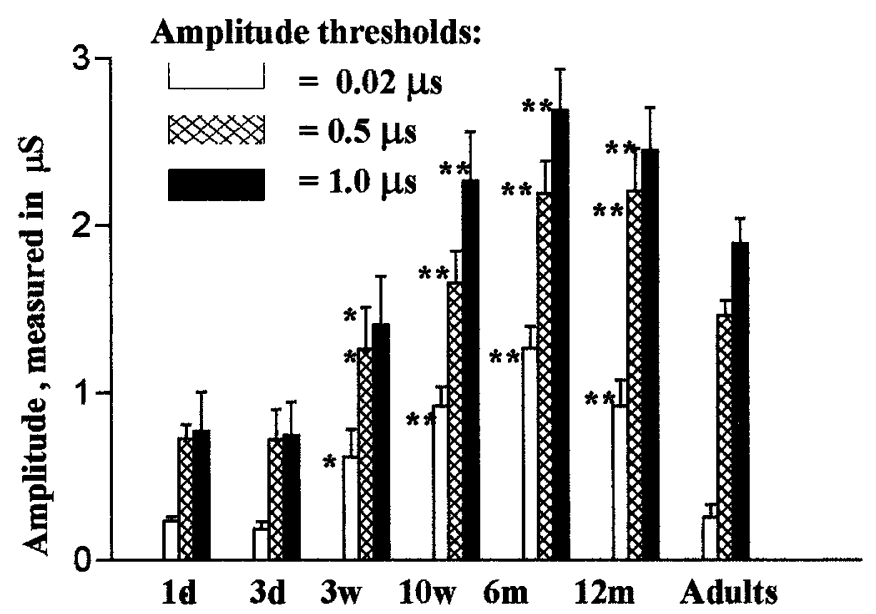

Figure 5. The increased amplitude of the waves during spontaneous skin conductance changes, using amplitude thresholds of $0.02,0.5$ and $1.0 \mu \mathrm{si}-$ emens $(* p<0.05)\left({ }^{* *} p<0.01\right)$.

also found $(p<0.001)$. The results from measurements 1,2 and 3 were different from the results from 4,5 , and 6 . At amplitude thresholds $0.02,0.5 \mathrm{~m}$ and $1.0 \mu$ siemens, the development of the amplitude during the 1st year was positively correlated to the infants' level of arousal $(p<0.001)$. When we corrected for the effect of time, arousal did not show any effect on the amplitude. More than $60 \mathrm{~min}$ since the last feed was associated with higher amplitude $(p<0.01)$.

The infant's sex and temperature, the method of delivery, use of delivery aid, and any anesthetics used during delivery had no statistically significant influence on any of the spontaneous skin conductance variables.

Stimulated SCC. The mean level of arousal $( \pm \mathrm{SD})$ before the onset of auditory stimuli was $2.04( \pm 0.91)$ during the first measurement, $2.5( \pm 0.95)$ during the second, $2.69( \pm 0.74)$ during the third, $2.85( \pm 0.60)$ during the fourth, $2.63( \pm 0.52)$ during the fifth and $2.45( \pm 0.59)$ during the last measurement.

During auditory stimulation the percentage of the infants that responded increased significantly during the first $10 \mathrm{wk}$ of life. Eight per cent responded on the 1st day of life and $63 \%$ responded at $12 \mathrm{mo}$ of life $(p<0.002)$. The result from measurement 1 was different from the results from the measurements 4,5 , and 6 , and results from measurements 2 and 3 were different from the results from measurement 4 (Table 1). (The adult value was $100 \%$.)

The mean amplitude of the response to the auditory stimulus increased from $1.43 \mu$ siemens during the 1st day of life up to $2.58 \mu$ siemens at $10 \mathrm{wk}$ of life $(p<0.02)$ (Table 1$)$. The result from measurement 1 was different from the results from measurement 4,5 , and 6 , and result from measurement 2 was different from the result from measurement 6 . (The adult value was $0.50 \mu$ siemens.)

No statistically significant change was found in the mean latency time (Table 1). The numerical value decreased from $2.1 \mathrm{~s}$ during the first measurement to $1.6 \mathrm{~s}$ at $6 \mathrm{mo}$, and then increased to $2.0 \mathrm{~s}$ at one year of life. (The adult value was $2.1 \mathrm{~s}$.)

No statistically significant change was found in the mean recovery time during the 1 st year. Only a small percentage recovered during this period of time $(20.3 \%)$. When individual responses were analyzed, signs of habituation were found in a few infants, but the habituation was not statistically significant.

\section{DISCUSSION}

During the first $10 \mathrm{wk}$ of life spontaneous SCC showed an increase in the mean skin conductance level, an increase in the number of waves per second with a higher amplitude threshold, and an increase in the wave amplitude $(p<0.001)$. During auditory stimulation the percentage of subjects that responded and the amplitude of the response both increased during the first 10 wk of life $(p<0.001)$, and then stabilized during the 1st year of life. No significant changes were found in the degree of habituation, the recovery or latency time. The latency time did not show a significant change during the 1st year of life, but its numerical value increased during the 1st year of life, and stabilized at approximately the same level as reported for an adult population (13).

Our results demonstrate that only $8.5 \%$ of the infants responded to the auditory stimuli during the 1 st week of life. Gladman et al. found that in response to a heel prick, the babies became aroused and that the level of mean skin conductance level rose sharply and immediately in 21 out of 22 babies (95\%) with a gestation age of 40-43 wk (14). This may indicate that our auditory stimulus was not as efficient as a heel prick in producing an SCC response. The heel prick response, measured by mean skin conductance level, increased during the 1st months of life in infants born to term (14).

Gladman also claimed that this increase is dependent on the innervating sympathetic nervous system, and on the properties of the sweat glands and the skin itself (14). Harpin et al. used a skin evaporation meter to detect the effect of increased arousal, and identified increased palmar water loss during the 1 st week of life (15). Likewise Rutter et al. stated that in term babies the water loss from the palms and soles measured in terms of the water vapor pressure gradient increased from birth

Table 1. The percentage of infants that responded to auditory stimuli, the amplitude of the response measured in $\mu$ s, as well as the latency time of the response

\begin{tabular}{cccccccc}
\hline & Day 1 & Day 3 & Week 3 & Week 10 & 6 Months & 12 Months & Adults \\
\hline \% of infants responded & 8.3 & 10.8 & $52^{* *}$ & $50^{* *}$ & $62^{* *}$ & $62^{* *}$ & 100 \\
Amplitude of response & $1.25(0.20)$ & $1.16(0.22)$ & $1.61(0.12)$ & $2.58(0.18)^{* *}$ & $2.62(0.20)^{* *}$ & $2.25(0.15)^{* *}$ & $0.50(0.09)$ \\
$\quad$ In $\mu$ siemens (2 SD) & & & & & & \\
Latency time in seconds & $2.09(0.19)$ & $1.92(0.14)$ & $1.93(0.09)$ & $1.84(0.07)$ & $1.63(0.06)$ & $1.19(0.06)$ & $1.44(0.008)$
\end{tabular}

$$
\text { (2 SD) }
$$

Stimulated skin conductance measurement at 1 and $3 \mathrm{~d}$ of life, at 3 and $10 \mathrm{wk}$ of age, as well as at 6 and 12 mo of age. Adult values are also recorded. $* * p<0.01$. 
up to the age of 4 weeks, and explained this increase by the onset of emotional sweating (16). The skin impedance, which is the "resistance" to an alternating current, has been shown to drop during the 1st postnatal months, and this is explained by increased skin hydration, again the product of greater functional maturity of the eccrine sweat glands (17). Emery states that the highest level of impedance is present at term, and then drops over the next 4 months (18). These results, like ours, all indicate that the mean skin conductance level increases, but they are unable to differentiate between the development of the palmar and plantar sweat glands themselves, the skin, and the development of the part of the sympathetic nervous system associated with arousal. Interestingly, the present study demonstrates that the number of waves with an amplitude threshold higher than $0.5 \mu$ siemens was influenced by arousal, when the influence of time had been corrected for. This finding indicates an increase in the activity of the part of the sympathetic nervous system associated with arousal during the first $10 \mathrm{wk}$ of life.

The sweat glands in the palms of the hand and soles of the feet are anatomically developed during the second trimester (17), but are not fully functional until 36 to $37 \mathrm{wk}$ of gestation (15). Joergensen et al. demonstrated that the emotional sweat glands were mature and functioning after 2 wk of life (19). Shankle found that the sweat gland duct length and the volume of the sweat gland coil both rise with age (20). These findings may indicate that the rise in amplitude and the mean skin conductance level in this study are also influenced by the maturity of the sweat glands.

Even though the peripheral sympathetic nervous system is developed at $18 \mathrm{wk}$ of gestation (21), myelinization continues to mature with increasing age (22). Littman et al. have demonstrated that both infants born to term and those born preterm show a significant correlation between nerve velocity and conceptional age (23). These results indicate that the maturation of the peripheral sympathetic nervous system may be a contributing factor.

During pregnancy the developing fetus relies primarily on the adrenal glands, the "nonneurogenic" response under stress, for the unselective discharge of catecholamine, but after a few weeks of life this direct response fades away as the sympathetic nerves and nerve endings develop and become functional (24, $25)$. In this nonneurogenic response, epinephrine predominates over norepinephrine (26), while during the neural response norepinephrine is predominant (25). These findings indicate that other part of the sympathetic nervous system continues to mature during the 1st weeks of life, which is in accordance with our results.

The fetal heart rate depends on a delicate balance between the sympathetic and parasympathetic nervous system. The development of the heart rate follows the development of SCC during the 1st months of life. In healthy infants the heart rate increases significantly from the 1 st week to the 3 rd month of life, and then returns to the neonatal rate and is stabilized at 6 months of age (27). On the other hand, the activity of the sympathetic nervous system that innervates the arteriovenous anastomoses in the skin, as measured by a laser Doppler flowmeter, does not increase during the first $14 \mathrm{wk}$ of life (28).
Measuring skin conductance is a safe, noninvasive and semitemperature-independent method, and it is also little influenced by circulatory changes $(29,30)$.

To conclude, the increase in the number of waves with a higher amplitude than $0.5 \mu$ siemens, the amplitude of the waves, and the mean skin conductance level indicate that the part of the sympathetic nervous system associated with arousal matures from birth up to the age of $10 \mathrm{wk}$. This method may be an effective way of evaluating stress in infants (31), and especially pain responses $(29,30)$. This method can also be used to evaluate impaired maturation of the sympathetic nervous system associated with arousal in connection with risk factors for the sudden infant death syndrome.

Acknowledgments. The authors thank Dr. Zhigui Qiao and Professor Sverre Grimnes for their assistance.

\section{REFERENCES}

1. Wallin BG 1978 Recordings of impulses in unmyelinated nerve fibres in man: sympathetic activity. Acta Anaesthesiol Scand Suppl 70:130-136

2. Esler MD, Lambert GW, Ferrier C, Kaye DM, Wallin BG, Kalff V, Kelly MJ, Jennings GL 1995 Central nervous system noradrenergic control of sympathetic outflow in normotensive and hypertensive humans. Clin Exp Hypertens 17:409-423

3. Macefield VG, Wallin BG 1996 The discharge behaviour of single sympathetic neurones supplying human sweat glands. J Auton Nerv Syst 14:277-286

4. Lindberg L, Wallin G 1981 Sympathetic skin nerve discharge in relation to amplitude of skin resistance response. Psychophysiology 18:268-270

5. Tranel D, Damasio H 1994 Neuroanatomical correlates of electrodermal skin conductance responses Psychophysiology 31:427-438

6. Qiao ZG, Morkrid L, Grimnes S 1987 Simultaneous measurement of electrical admittance, blood flow and temperature at the same skin site with a specially designed probe. Med Biol Eng Comput 25:299-304

7. Grimnes S 1982 Psychogalvanic reflex and changes in electrical parameters of dry skin. Med Biol Eng Compute 20:734-740

8. Grimnes S 1984 Pathways of ionic flow through human skin in vivo. Acta Derm Venereol 64:93-98

9. Martinsen G, Grimnes S, Sveen O 1997 Dielectric properties of some keratinized tissues. Part 1: Stratum corneum and nail in situ. Med Biol Eng Comput 35:172-176

10. Grimnes S 1983 Impedance measurements of individual skin surface electrodes. Med Biol Eng Comput 21:750-755

11. Storm H, Fremming A, Odegaard S, Marthinsen OG, Morkrid L 2000 The development of a software program for analyzing spontaneous and externally elicited skin conductance changes in infants and adults. Clin Neurophysiol 111:1889-1898

12. Edelberg R 1967 Electrical properties of the skin. In: Brown CC (eds), Methods in Psychophysiology. Williams \& Wilkins, Baltimore, pp 1-59

13. Gutrecht JA 1994 Sympathetic skin response. J Clin Neurophysiol 11:519-524

14. Gladman G, Chiswick ML 1990 Skin conductance and arousal in newborn. Arch Dis Child 65:1063-1066

15. Harpin VA, Rutter N 1982 Development of emotional sweating in the new-born infant. Arch Dis Child 57:691-695

16. Rutter N, Hull D 1979 Water loss from the skin of term and preterm babies. Arch Dis Child 54:858-868

17. Mize MM, Aguirre Vila-Cord A, Prager TC 1989 The relationship between postnatal skin maturation and electrical skin impedance. Arch Dermatol 125:647-650

18. Emery MM, Hebert AA, Prager TC 1991 The relationship between skin maturation and electrical skin impedance. J Dermatol Sci 2:336-340

19. Jorgensen RJ, Salinas CF, Dowben JS, St John DL 1988 A population study on the density of palmar sweat pores. Birth Defects Orig Artic Ser 24:51-63

20. Shankle WR, Azen SP, Landing BH 1982 Comparisons of eccrine sweat gland anatomy in genetic, chromosomal, and other diseases, and a suggested procedure for use of sweat gland measurements in differential diagnosis. Teratology 25:239245

21. Ellis RA 1968 Eccrine sweat glands: electron microscopy, cytochemistry and anatomy . In: Jadassohn J (ed) Handbuch der Haut-und Geschlechtskrankheiten, Vol 1. Springer Verlag, Berlin, pp 224-266

22. Pamphlett R, Murray N, Louda C 1996 Phrenic nerve maturation in the sudden infant death syndrome. Acta Neuropathol 91:422-426

23. Littman B 1975 Peripheral nerve maturation in premature infants. Neuropadiatrie 6:284-291

24. Lagercrantz H, Slotkin TA 1986 The "stress" of being born. Sci Am 254:100-107

25. Slotkin A, Seidler FJ, 1988 Adrenomedullary catecholamine release in the fetus and newborn. J Dev Physiol 10:1-16

26. Rachel L, Copper R, Goldenberg R 1990 Catecholamine secretion in fetal adaptation to stress. J Obstet Gynecol Neonatal Nurs 19:223-226 
27. Schwartz PJ, Culbertson JK, Krous HF, Bendell RD 1989 The cardiac theory and sudden infant death syndrome. In: Arnold E (ed) Sudden Infant Death Syndrome. Medical Aspects and Psychological Management. Johns Hopkins University Press, Baltimore, pp 121-138

28. Lossius K, Eriksen M 1994 Connection between skin arteriovenous shun flow fluctuations and heart rate variability in infants. Early Hum Dev 39:69-82
29. Wallin GB, Sundlof G, Delius W 1975 The effects of carotid sinus nerve stimulation on muscle and skin nerve sympathetic activity in man. Pflugers Arch 358:101-110

30. Hagbarth KE, Hallin RG, Hongell A, Torebjørk HE, Wallin BG 1971 General characteristics of sympathetic activity in human skin nerves Acta Physiol Scand $84: 164-176$

31. Storm H 2000 Skin conductance and the stress response from heel stick in preterm infants. Arch Dis Child Fetal Neonatal Ed 83:F143-147 